\title{
T2 mapping vs T2 weighted imaging in the detection of myocardial oedema
}

\author{
Elisa McAlindon ${ }^{1 *}$, Peter Weale ${ }^{2}$, Jessica Harris ${ }^{1}$, David Smith ${ }^{1}$, Andreas Baumbach¹, Tom Johnson', \\ Chiara Bucciarelli-Ducci ${ }^{1}$ \\ From 15th Annual SCMR Scientific Sessions \\ Orlando, FL, USA. 2-5 February 2012
}

\section{Background}

The "gold standard" CMR sequence for assessing the myocardial oedema or area at risk following an acute coronary syndrome is controversial. T2 Short Tau Inversion Recovery (T2-STIR) is in widespread clinical use but can lack robustness. Steady state free precession oedema imaging (SSFP/ ACUT2E) has emerging data to support it as a more reproducible method for area at risk (AAR) assessment. We tested a novel T2 mapping method to AAR. The potential benefit of this method is that the numerical output of the method is largely independent of myocardial motion, instrumental errors (eg surface coil normalisation methods). The aim of this study was to compare the novel T2 mapping method with the two existing methods of assessing AAR (T2STIR and SSFP/ACUT2E).

\section{Methods}

30 slices in 10 patients day 2-4 following acute myocardial infarction were analysed by 3 sequences (T2-STIR, ACUT2E, and T2 mapping). The images were analysed using a semi-automated software, and the AAR was expressed as a \% of total slice area. The window setting was defined as the sum of the mean signal intensity (SI) of the unaffected area plus 2 standard deviation (SD) for this area. The level setting was set at the mean SI of the unaffected area (a method used in previous published studies). Inter-method and inter-observer variability was assessed using the Bland Altman method. Qualitative inter-observer and inter-method variability was assessed: each slice split into segments according to the 17 segment model and oedema in each segment scored as present of absent.

\section{Results}

On the Bland Altman plots we observed a better agreement between for T2-STIR vs T2 map, than ACUT2E vs T2 map (Image 1).

On qualitative assessment, there is very good agreement between T2-STIR and T2 map (kappa 0.71, 86\% segments agree) and ACUT2E and T2 map (kappa 0.81, $91 \%$ segments agree). We also found a good agreement between T2-STIR and ACUT2E (kappa 0.78, 89\% segments agree).

On assessing qualitative inter-observer reproducibility, there is a good agreement between observers using all 3 sequences; T2-STIR appears to have the lowest interobserver agreement (T2-STIR kappa 0.56, ACUT2E kappa 0.67, T2 map kappa 0.67).

\section{Conclusions}

T2 mapping method may provide a viable alternative to current AAR methods. This needs to be further assessed in a larger patient population.

\section{Funding}

NIHR Cardiovascular BRU, Bristol Heart Institute.

\section{Author details}

${ }^{1}$ Bristol Heart Institute, NIHR Cardiovascular BRU, Bristol, UK. ${ }^{2}$ Siemens

Medical Solutions, London, UK.

Published: 1 February 2012

doi:10.1186/1532-429X-14-S1-P266

Cite this article as: McAlindon et al:: T2 mapping vs T2 weighted

imaging in the detection of myocardial oedema. Journal of

Cardiovascular Magnetic Resonance 2012 14(Suppl 1):P266.

${ }^{1}$ Bristol Heart Institute, NIHR Cardiovascular BRU, Bristol, UK

Full list of author information is available at the end of the article

(c) 2012 McAlindon et al; licensee BioMed Central Ltd. This is an open access article distributed under the terms of the Creative 

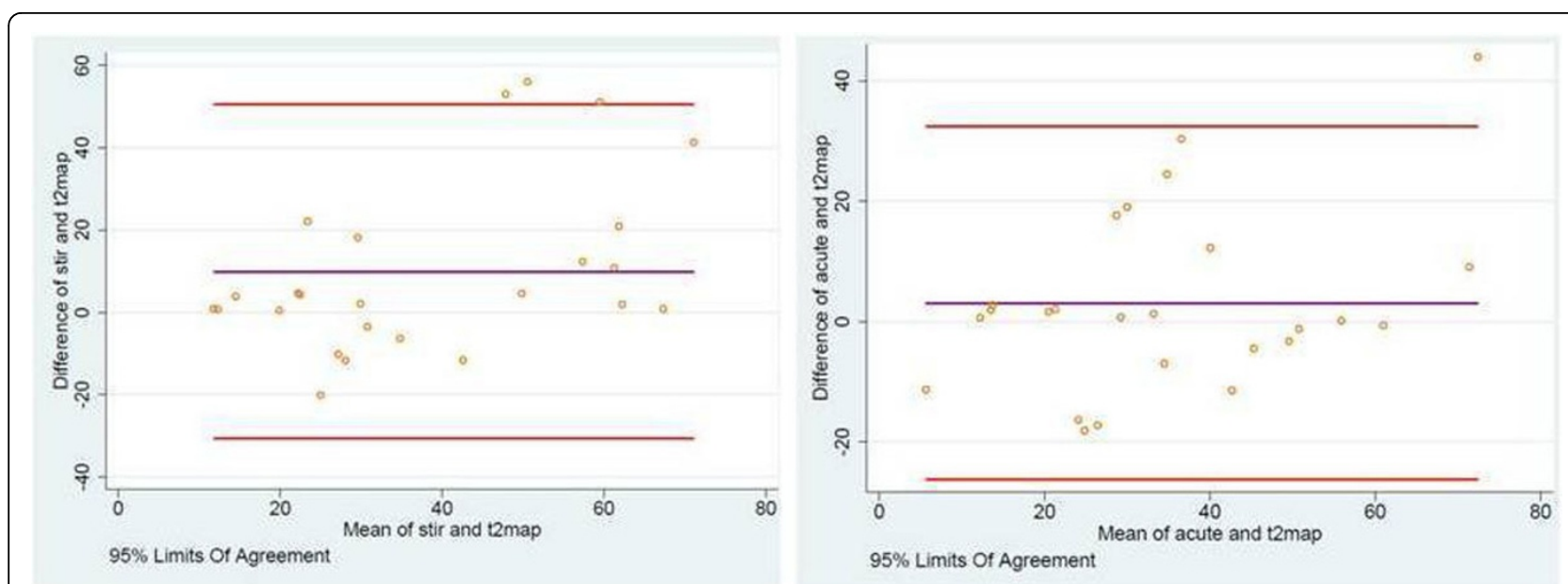

Figure 1 Bland Altman plots for AAR assessed by T2 mapping (t2map) vs STIR (left panel) and SSFP (acute)(right B).

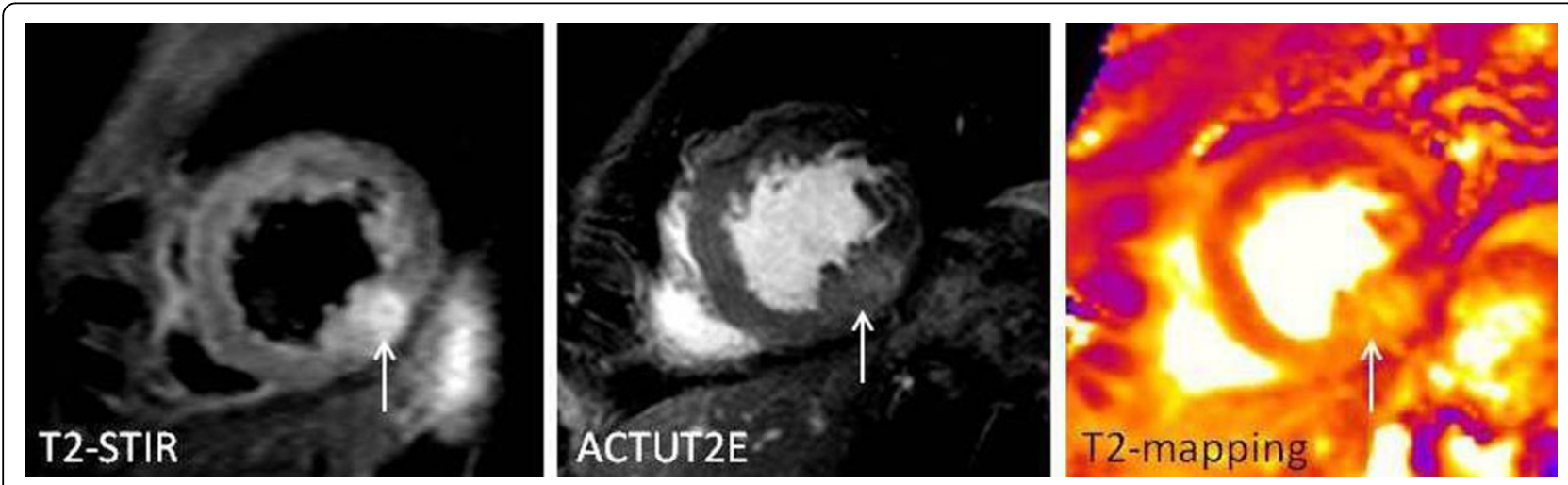

Figure 2 Short-axis slice repeated with the 3 techniques showing an oedematous area in the apical inferior wall. 